Part 2 Cosmic Dawn and Eoch of Reionization: observations, challanges and the first results 


\title{
Current Status of the LOFAR EoR Key Science Project
}

\author{
L. V. E. Koopmans ${ }^{1}$, on behalf of the LOFAR EoR KSP team \\ ${ }^{1}$ Kapteyn Astronomical Institute, University of Groningen, P.O.Box 800, NL-9700AV, \\ Groningen, the Netherlands
}

\begin{abstract}
A short status update on the LOFAR Epoch of Reionization (EoR) Key Science Project (KSP) is given, regarding data acquisition, data processing and analysis, and current power-spectrum limits on the redshifted 21-cm signal of neutral hydrogen at redshifts $z=8-10$. With caution, we present a preliminary astrophysical analysis of $\sim 60 \mathrm{hr}$ of processed LOFAR data and their resulting power spectrum, showing that potentially already interesting limits on $\mathrm{X}$-ray heating during the Cosmic Dawn can already be gained. This is by no means the final analysis of this sub-set of data, but illustrates the future potential when all nearly $3000 \mathrm{hr}$ of data in hand on two EoR windows will have been processed.
\end{abstract}

Keywords. Cosmology, Reionization, Cosmic Dawn

\section{Introduction}

Detecting the 21-cm signal of neutral hydrogen from the Epoch of Reionization (EoR), let alone the Cosmic Dawn (CD), is a very daunting task. A signal detection and its subsequent characterization, however, will scientifically be extremely rewarding. It will revolutionize our understanding of many astrophysical processes that occurred during the formation of the first stars, galaxies, and super-massive black holes, as well as the enrichment of the inter-galactic medium (IGM). It would also provide a "motion picture" of the infant Universe spanning a nearly one billion year time frame, opposed to the single "snapshot" available to us from the Cosmic Microwave Background (CMB), and form the foundation of our understanding of the present-day Universe. Intermingled with information about astrophysical processes occurring during these early phases, information about e.g. cosmology and dark matter can also be gained from understanding the spatial and temporal changes in the 21-cm signal, which especially on larger scales are still coupled to the underlying dark-matter distribution. Astrophysical effects such as ionisation and spin-temperature changes, however, need to be accounted for. Currently there are a number of experiments/projects (PAPER, MWA, LOFAR and the GMRT) that aim to detect an characterize the 21-cm signal of neutral hydrogen, in particular from the EoR. The CD - although within the frequency band of several of these telescopes - remains out of reach to due a lack of sensitivity. Smaller-scale experiments are ongoing with LWA/LEDA, and soon with NenuFar, as well as past experiments with 21CMA. Global signal experiment (e.g. EDGES and SARAS) aim to measure the average 21-cm signal, rather than it spatial fluctuations. Many of these experiments use different types and layouts of the radio array (or single receiver). All, apart from the GMRT which is dish-based, are aperture arrays. In the remainder, however, we focus on results from LOFAR. 


\section{Project Status}

In this section we shortly describe the LOFAR instruments, where related to EoR science, data acquisition, processing and analysis, as well as the first published results and more recently (unpublished) results from the analysis of five nights of data.

\subsection{The LOFAR Telescope}

The Low Frequency Array (LOFAR) consists of two distinct aperture arrays: the LowBand Antenna (LBA) system (typically $30-80 \mathrm{MHz}$ ) and the High-Band Antenna (HBA) system (110-240 MHz). Only the latter has sufficient sensitivity to detect the 21-cm signal of neutral hydrogen and hence it is the prime instrument used by the LOFAR EoR KSP team. LOFAR HBA consists of 48 core stations and 13 remote stations spread over the the Netherlands. The Dutch stations consist of 24 operational tiles each consisting of $4 \times 4$ cross-dipoles. The maximum single station-beam bandwidth is $96 \mathrm{MHz}$. This bandwidth can be traded against number of beams, which later in our observing campaign has been utilized. Baselines cover roughly the range of $35 \mathrm{~m}$ to $1000+\mathrm{km}$ (to LOFAR stations in Europe). Most collecting area and 21-cm signal brightness temperature sensitivity comes from the core region of about $3 \mathrm{~km}$ diameter width. A detailed description of LOFAR can be found in van Haarlem et al. (2013).

\subsection{Observations}

Although the LOFAR EoR KSP team targets two main windows, centred on 3C196 and the NCP, respectively, here we only present results from the NCP field. Including the current LOFAR observing Cycle, about 2000 hrs of data will have been acquired on the NCP by the spring of 2018, spread over about a hundred and fifty nights between 2012 and 2018. During later LOFAR observing cycles, multiple beams (currently up to seven) were used covering a wider area on/around the NCP over a more restricted bandwidth. Here we present only an analysis from single-beam observations in the $115-189 \mathrm{MHz}$ range. Raw visibilities from all baselines are typically acquired with $2 \mathrm{sec}$ and $3 \mathrm{kHz}$ correlator settings. These are down averaged, after initial pre-processing and flagging, to 15 and $60 \mathrm{kHz}$, respectively (retaining the time resolution). We store the highest resolution data for later use in the LOFAR long term archive, and currently only analyse the $60 \mathrm{kHz}$ channel data. The latter data are stored on our GPU-based processing cluster ( "Dawn"), which has a compute capability of 124 NVIDIA K40 GPUs and about 5 PB storage capacity. Each of the $2 \mathrm{~s}-60 \mathrm{kHz}$ down averaged data sets is several terabyte (TB) in volume, which grows by about a factor of 2.5 during processing. A more detailed description of the observations are given in Patil et al. (2017).

\subsection{Data Processing}

Data processing consist of several steps, being pre-processing, flagging, data averaging, direction independent and direction dependent calibration, imaging, foreground removal and power-spectrum construction (Patil et al. (2017)). We will not discuss these in great detail but refer to the relevant papers where necessary.

\subsubsection{Preprocessing and Flagging}

All raw data sets are preprocessed using NDPPP, which nowadays also includes flagging with AOFlagger, and if preferred also some form of direction independent calibration. We do the latter independently (see below). Stations with known problems are flagged and, conservatively, also baselines with a common electronics cabinet, which might suffer from mutual coupling. After flagging the data is averaged to a lower spectral (and in some cases temporal) resolution and used in processing. 

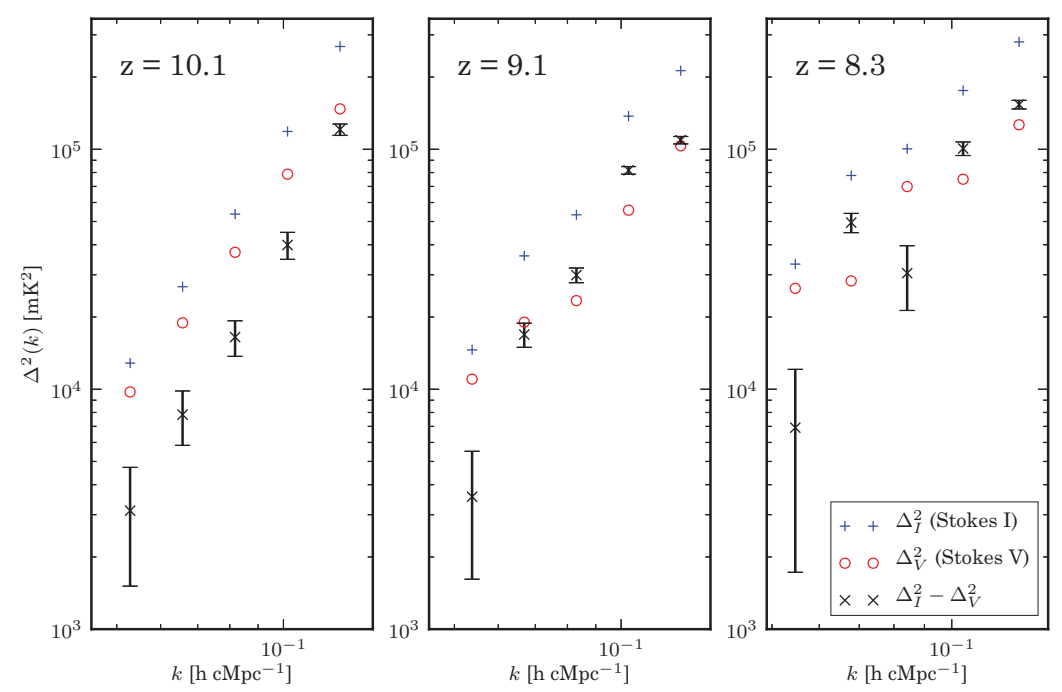

Figure 1. The spherically averaged Stokes I and V power spectra after foreground removal using GMCA. The mean redshifts are indicated in the panels. See Patil et al. (2017)

\subsubsection{Calibration}

Once the data has been pre-processed, it is calibrated using on a "sky-model", which has been built via an iterative process during the KSP observing campaign and is continuously improved. It currently consists of about 21,000 components, each with their own spectral shape. The current model also includes a diffuse components in Stokes I, Q and $\mathrm{U}$ in order to avoid calibration biases on the short baselines (below about $250 \lambda$ ). Data is initially only corrected for the four directionally-independent gains in the station-based Jones matrix. Calibration is simultaneously done over the full frequency band using consensus optimization in SageCal-CO (Yatawatta (2015)) to maximize smoothness over the frequency domain and still allow for individual solutions per $60 \mathrm{kHz}$ frequency channel. These solutions are applied to the data such that both structure in the sky-model and structure not in the sky-model (e.g. faint compact and diffuse emission) will have these solutions applied to them. During the second calibration step the sky-model components are combined in about 122 clusters spread over about 40 degrees field of view. Most of these clusters are inside the inner primary beam area, with a typical size of 1-2 degrees, but also include the brighter sources outside the beam, as well as dominant sources very far away (CasA, CygA, etc.). A simultaneous (i.e. direction-dependent) solution is obtained for the gains of all 122 clusters and a single direction-dependent solution for the diffuse sky model (to limit the computational demand). The sky-model of 21,000 sources is subsequently subtracted from the data with their gain solutions applied (the same gain is applied to all components inside one cluster). The residual visibilities are stored for further processing.

\subsubsection{Imaging}

The field of view of the LOFAR-HBA station beam is nearly 4 degrees at $150 \mathrm{MHz}$, and images with resolutions as high as 6 arcsec are constructed using all Dutch baselines over about 40 degree field of view in order to built the very detailed calibration models of the sky discussed above. European baselines are included to construct calibration models of the brightest sources in the field. Data cubes over $3 \times 3$ degrees are made with a spectral resolution currently up to $60 \mathrm{kHz}$ and about 10 arcminute spatial resolution. These are 

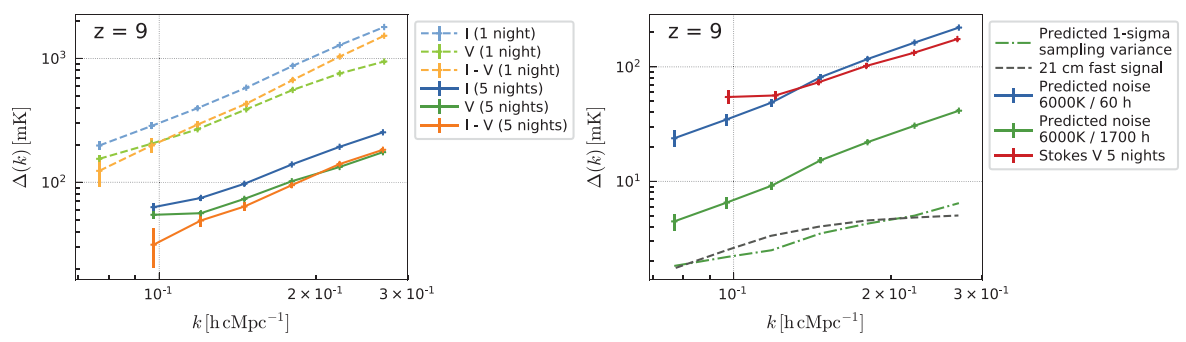

Figure 2. The spherically averaged Stokes I and V power spectra after SageCal-CO plus GPR at $z=9$. Left: the solid lines show the power spectra after five night nights for Stokes I and V and their difference. The dashed lines are for a single night as published in Patil et al. (2017). The improvement is quite striking being nearly 2 orders of magnitude in power. Right: Forecast from the current limits to the full LOFAR EoR data sets.

used for further 21-cm signal analyses. Given that the latter images are only produced after direction dependent calibration, they do no longer include the 21,000 source components that were subtracted. They do include direction independent calibrated diffuse emission in all Stokes parameters though, and residual source structure that were not part of the sky model.

\subsubsection{Removal Diffuse Emission}

The final step before analysing the residuals is to remove the diffuse emission from the Galactic foregrounds. In Patil et al. (2017) we used the GMCA method to accomplish this (Chapman et al. (2013)), which removes the dominant components from the datacube (spatial and frequency). More recently however we are using Gaussian Process Regression (GPR; Mertenset al. (2018)) which allows each of the foreground components to be defined via a Gaussian Process with its own covariance matrix. The latter has itself a set of free parameters which are optimised for via a Bayesian analysed. This has allows for a much better foreground removal, shown both in simulations and in real LOFAR data.

\subsection{Results and new developments since 2017}

As was clear from the analysis of the data in Patil et al. (2017), the thermal noise level at that moment could not be reached in the power-spectra in Stokes I (see Fig. 1), and neither in Stokes Q, U and V. Our current understanding is that this results from the calibration process, where structure in the sky is not accounted for in the calibration model. The resulting gain errors (direction dependent) are transfer to the smaller baselines and cause excess noise in the power spectrum (Patil et al. (2016)). By enforcing both spectral smoothness in the gain solution of SageCal-CO and accounting for residual spectral structure in the GPR, we are now able to reach the thermal noise to within 10$20 \%$. In Fig. 2 a very preliminary power-spectrum is shown based on five analysed data sets, showing a dramatic improvement in depth. Similar results are obtained at redshifts $z=8$ and 10 as well (not shown). The same Figure also shows that a nominal 21-cm EoR signal can be detected in about $1700 \mathrm{hr}$ of NCP data, roughly equivalent to the data in hand.

\subsubsection{Astrophysical Analysis}

Using the preliminary results from these five analysed and combined power-spectra, we have analysed these using the latest version of the code 21CMMC (Greig \& Mesinger (2015)), which includes X-ray heating, Lyman alpha coupling, spin-temperature and recombination effects. Since the current power-spectra are formally still "detections" of 


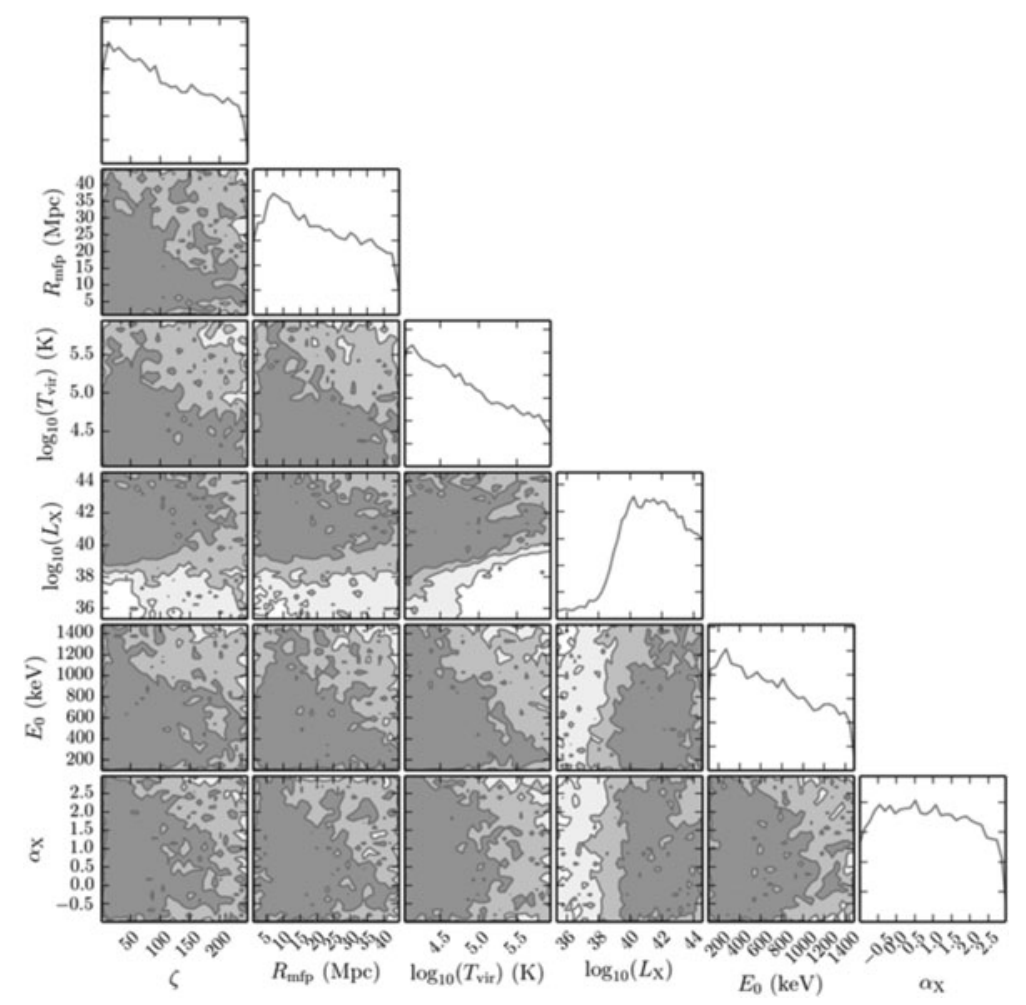

Figure 3. Preliminary constraints set on the six driving parameters of the Cosmic Dawn and EoR using 21CMMC and five night of LOFAR EoR data.

some minor excess noise, which we believe are (incoherent) residuals from the calibration process (see above), this analysis should be seen as first attempt. To address this excess, we conservatively double the errors and set the expectation values of the upper-limit power spectra to zero (i.e. the PDF is a one-sided Gaussian). Given that the expected 21-cm signal is very much below the current limits this is a perfectly reasonable and conservative approach. We also combine the power-spectra at redshifts $z=8-10$ and at all $k$-modes in the analysis. The results of the MCMC run are shown in Fig. 3. Most parameters are still poorly constrained based on five night of data, but the X-ray luminosity per unit SFR is already constrained from below. Effectively a cold IGM model is being limited by the upper limit of the 21-cm power-spectrum at $z=8-10$. In short, for very cold IGM models the predicted power-spectra would already exceed the observed 21-cm signal limits of LOFAR after five nights of integration.

\section{Conclusion and Future Work}

Since 2012 the LOFAR EoR KSP team has collected nearly $3000 \mathrm{hr}$ of observational data with LOFAR-HBA, accruing $5 \mathrm{~PB}$ of frequency-averaged $(2 \mathrm{sec}-60 \mathrm{kHz})$ visibilities on two fields: the NCP and 3C196. We are still observing. In parallel to this observational effort, we have put considerable effort in to improving our direction-dependent calibration, wide-field imaging and diffuse foreground removal algorithms, as well as deepening our understanding of the signal processing chain. We have recently upgraded our GPUbased computing and storage cluster and have improved the GPU processing speed by factors of about ten since 2010. These accumulated improvements and acceleration in 
the data processing are allowing us to process the data via a nearly automated pipeline to a level that for the first time closely approaches the thermal noise per night. The "excess variance" (i.e. variance far exceeding the thermal noise level) that was previously seen in the residual data has largely been mitigated via (i) much better regularised gain solutions (i.e. with SageCal-CO), by (ii) including diffuse models in the calibration process in Stokes I, Q and U, and by (iii) using a GPR method in the diffuse and residual foreground removal. The current focus of the team is to further understand any potential "bias" that might suppress (or enhance) the 21-cm signal. We do this via injected signals (Mevius et al. in prep). Such bias analyses are crucial to build confidence in the results, but are very complicated, given that the $21-\mathrm{cm}$ is far below the current thermal noise and the effect could be signal-strength and scale dependent. Current results show largely incoherent residual power-spectra (assessed via their cross power spectra), allowing the data to be averaged. The noise level appears to decrease as it should for uncorrelated residuals, at least for the first five nights. All this bodes well, but is still no guarantee that this averaging will continue down to the 21-cm signal. Effects of residual sky model effect, the ionosphere, polarisation leakage, signal processing effects, etc. might still require improved data processing schemes in the future. Preliminary 21-cm power spectrum limits at $z=8-10$, obtained after five nights, however, show that current LOFAR result are able to limit cold IGM models at these high redshift, putting lower limits on the heating of the IGM during the Cosmic Dawn. However, until a complete bias and variance analysis have been completed, these results should be taken with caution.

\section{Acknowledgement}

In memory of Ger de Bruyn who passed away in July 2017, but is still inspiring us. We thank the organisers of the symposium for financial support.

\section{References}

Chapman, E., Abdalla, F. B., Bobin, J., Starck, J., Harker, G., Jelić, V., et al. (2013). The scale of the problem: recovering images of reionization with Generalized Morphological Component Analysis. Monthly Notices of the Royal Astronomical Society, 429(1), 165-176. http://doi.org/10.1093/mnras/sts333

Greig, B., \& Mesinger, A. (2015). 21CMMC: an MCMC analysis tool enabling astrophysical parameter studies of the cosmic $21 \mathrm{~cm}$ signal. Monthly Notices of the Royal Astronomical Society, 449(4), 4246-4263. http://doi.org/10.1093/mnras/stv571

van Haarlem, M. P., Wise, M. W., Gunst, A., Heald, G., McKean, J. P., Hessels, J. W. T., et al. (2013). LOFAR: The LOw-Frequency ARray. Astronomy and Astrophysics, 556, A2. http://doi.org/10.1051/0004-6361/201220873

Mertens, F. G., Ghosh, A., \& Koopmans, L. V. E. (2017, November 29). Statistical 21-cm Signal Separation via Gaussian Process Regression Analysis. arXiv.org.

Patil, A. H., Yatawatta, S., Zaroubi, S., Koopmans, L. V. E., de Bruyn, A. G., Ciardi, B., et al. (2016, May 24). Systematic biases in low frequency radio interferometric data due to calibration: the LOFAR EoR case. arXiv.org. http://doi.org/10.1093/mnras/stw2277

Patil, A. H., Yatawatta, S., Koopmans, L. V. E., de Bruyn, A. G., Brentjens, M. A., Zaroubi, S., et al. (2017). Upper Limits on the $21 \mathrm{~cm}$ Epoch of Reionization Power Spectrum from One Night with LOFAR. The Astrophysical Journal, 838(1), 65. http://doi.org/10.3847/1538$4357 /$ aa63e7

Yatawatta, S. (2015). Distributed radio interferometric calibration. Monthly Notices of the Royal Astronomical Society, 449(4), 4506-4514. http://doi.org/10.1093/mnras/stv596 